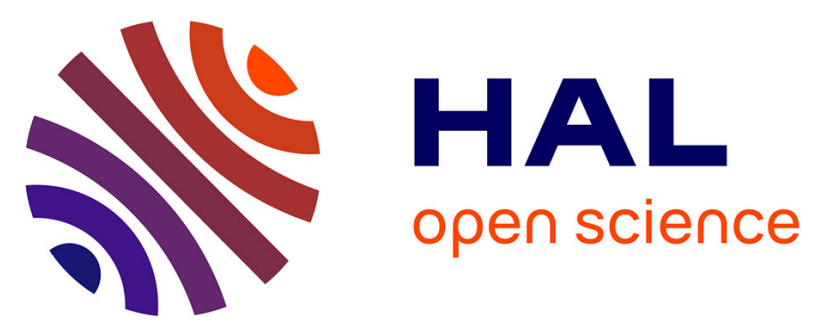

\title{
Impact d'une campagne de sensibilisation sur le cancer sur un échantillon de femmes en traitement pour un premier cancer du sein
}

\author{
M. Barrault, G. Michel, F. Cousson-Gélie
}

\section{- To cite this version:}

M. Barrault, G. Michel, F. Cousson-Gélie. Impact d'une campagne de sensibilisation sur le cancer sur un échantillon de femmes en traitement pour un premier cancer du sein. Annales MédicoPsychologiques, Revue Psychiatrique, 2010, 168 (6), pp.466. 10.1016/j.amp.2010.04.017. hal00659517

\section{HAL Id: hal-00659517 https://hal.science/hal-00659517}

Submitted on 13 Jan 2012

HAL is a multi-disciplinary open access archive for the deposit and dissemination of scientific research documents, whether they are published or not. The documents may come from teaching and research institutions in France or abroad, or from public or private research centers.
L'archive ouverte pluridisciplinaire HAL, est destinée au dépôt et à la diffusion de documents scientifiques de niveau recherche, publiés ou non, émanant des établissements d'enseignement et de recherche français ou étrangers, des laboratoires publics ou privés. 


\section{Accepted Manuscript}

Title: Impact d'une campagne de sensibilisation sur le cancer sur un échantillon de femmes en traitement pour un premier cancer du sein

Authors: M. Barrault, G. Michel, F. Cousson-Gélie

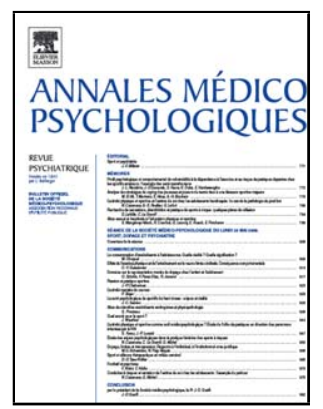

PII:

S0003-4487(10)00144-7

DOI: doi:10.1016/j.amp.2010.04.017

Reference: $\quad$ AMEPSY 1162

To appear in: $\quad$ Annales Médico-Psychologiques

Please cite this article as: Barrault M, Michel G, Cousson-Gélie F, Impact d'une campagne de sensibilisation sur le cancer sur un échantillon de femmes en traitement pour un premier cancer du sein, Annales medio-psychologiques (2008), doi:10.1016/j.amp.2010.04.017

This is a PDF file of an unedited manuscript that has been accepted for publication. As a service to our customers we are providing this early version of the manuscript. The manuscript will undergo copyediting, typesetting, and review of the resulting proof before it is published in its final form. Please note that during the production process errors may be discovered which could affect the content, and all legal disclaimers that apply to the journal pertain. 


\title{
Communication
}

Impact d'une campagne de sensibilisation sur le cancer sur un échantillon de femmes en traitement pour un premier cancer du sein

\section{Impact of a national cancer awareness campaign in primary breast cancer patients}

\author{
M. Barrault ${ }^{\text {a }}$, G. Michel ${ }^{\text {b }}$, F. Cousson-Gélie ${ }^{\text {b }}$ \\ ${ }^{a}$ Institut Bergonié, Unité de Psychologie Clinique, 229, Cours de l'Argonne \\ 33076 Bordeaux Cedex, France \\ ${ }^{b}$ Université Victor Segalen Bordeaux 2, Laboratoire de Psychologie Santé et Qualité de vie \\ EA4139, $3^{\text {ter }}$, Place de la Victoire, 33076 Bordeaux Cedex, France
}

Auteur correspondant: Mme Marion Barrault, Institut Bergonié, Unité de Psychologie Clinique, 229, Cours de l'Argonne, 33076 Bordeaux Cedex, France

Tél. : 0556333294

Fax : 0556337819

Adresse email : barrault@bergonie.org 


\title{
Résumé
}

En 2007, l'Institut National du Cancer a initié un programme d'information intitulé «Nous sommes deux millions de héros ordinaires». Les perceptions concernant cette campagne ont été analysées chez 34 femmes traitées pour un premier cancer du sein via des entretiens qualitatifs et l'évaluation de certaines variables psychologiques. Trois processus identificatoires ont été dégagés :

- une identification à la figure du héros avec une valorisation de la répression émotionnelle ;

- une adhésion au contenu « ordinaire » avec un faible sentiment d'efficacité perçue ;

- une identification impossible en réponse à l'injonction paradoxale.

Ces résultats confirment la nécessité de promouvoir des campagnes de sensibilisation prenant en compte les effets possibles du discours médiatique sur les individus aux prises avec la maladie cancéreuse.

Mots clés: Analyse qualitative; Campagne d'information; Cancer du sein; Détresse émotionnelle ; Stratégies de coping

\begin{abstract}
In 2007, the French National Cancer Institute initiated a cancer communication campaign entitled "We are two million ordinary heroes". Aims of this study were to investigate perceptions about this campaign, for 34 women treated for breast cancer. Triangulation of the reports from narratives and assessment of psychological variables ensures better validity of the data. Three psychological identification processes have been identified:

- identification with the hero character with a value of emotional repression;

- adherence to content "ordinary" with low level of perceived self-efficacy;

- none identification in response to a double-bind message, with sense-making process about traumatic event.

These findings underscore the need to promote communication health campaigns taking into account possible effects of media discourse on individuals and families struggling with cancer.
\end{abstract}

Keywords: Breast cancer; Communication campaign; Coping strategies; Emotional distress; Qualitative analysis 


\section{Introduction}

\subsection{Maladie cancéreuse, stratégies d'adaptation et détresse psychologique}

Avec 49814 nouveaux cas estimés en 2005 en France, le cancer du sein reste le premier cancer chez les femmes [13]. En 2009, plus de $70 \%$ des femmes atteintes d'un cancer du sein survivront au moins 15 ans après le diagnostic, et près de $90 \%$ au moins cinq ans [3]. Malgré les évolutions thérapeutiques et la diminution de la mortalité, un diagnostic de cancer demeure un événement de vie majeur pour la plupart des femmes [21]. Le bouleversement émotionnel généré par l'apparition de la maladie peut se traduire par l'émergence de détresse psychologique $[9,17,20,24]$. Ce sont les capacités d'adaptation de chaque individu face à la maladie qui détermineront notamment l'intensité et le devenir de la détresse émotionnelle [9]. Ainsi, l'évitement ou la répression émotionnelle tendent à prédire une qualité de vie altérée et plus de détresse psychologique [9,12,21]. D'autre part, les stratégies centrées sur la résolution de problèmes ou de la réévaluation positive sont positivement liées à plusieurs aspects du bien-être subjectif et de la qualité de vie [6,22].

\subsection{Représentations sociales et cancer}

Malgré les progrès thérapeutiques, l'évaluation des programmes d'éducation à la santé souligne la persistance de représentations péjoratives liées au cancer. Ainsi, les idées de mort, incurabilité, de maladie honteuse qu'il serait préférable de cacher à son entourage continuent à occuper une place prépondérante chez les individus interrogés, à l'évocation de la maladie cancéreuse [2,5,10]. Avec l'objectif de faire évoluer ces représentations, l'Institut National du Cancer a initié en janvier 2007 un programme d'information intitulé « Nous sommes deux millions de héros ordinaires » (diffusion de spots télévisuels de témoignages d'individus en rémission) [14]. L'expression «héros ordinaires » a été choisie à dessein et s'inspire du mouvement communautaire américain des «long term survivors » [16].

\subsection{Objectifs de l'étude}

Compte tenu des rares études sur le sujet, il a paru intéressant d'explorer la perception de ce type de campagne de communication chez un échantillon de patientes traitées pour un 
premier cancer du sein, dans les quatre premiers mois suivant l'annonce de la maladie, et d'articuler ce discours avec certaines variables psychologiques.

\section{Méthodologie}

\subsection{Population étudiée}

Cette étude a été menée dans un service de chirurgie oncologique, auprès de 132 femmes devant subir un acte chirurgical pour un premier cancer du sein. Parmi elles, 37 ont participé à des entretiens qualitatifs. Cette étude porte plus particulièrement sur ce sous-groupe. Les participantes étaient âgées de 31 à 76 ans $(M=54,87 \pm 11,45)$ et ont toutes signé un consentement éclairé. L'ensemble des données sociodémographiques et médicales sont présentées dans le tableau 1. Le sous-groupe n'est pas différent de l'ensemble de l'échantillon au plan de l'âge $(\mathrm{t}(130)=0.21)$.

\subsection{Déroulement de l'étude}

Pendant l'hospitalisation pour l'opération chirurgicale (T0), les traits de personnalité, la qualité de vie, la détresse émotionnelle ont pu être évalués par questionnaires. Un mois après l'acte chirurgical (T1), la détresse émotionnelle, la qualité de vie et les stratégies de coping ont été évaluées, et les patientes ayant donné leur accord ont rencontré un psychologue pour un entretien semi-directif. Quatre mois après la chirurgie (T2), un courrier comportant les mêmes questionnaires a été envoyé à l'ensemble des patientes.

\section{3. Évaluation de la perception de la campagne d'information}

Au cours d'un entretien, nous avons introduit la question suivante : «Avez-vous vu la campagne de l'Institut National du Cancer : Nous sommes deux millions de héros ordinaires? Si oui, qu'en pensez-vous ?»

Notre position paradigmatique se situe entre les paradigmes post-positivistes et interprétatifs-constructivistes [15]. L'analyse des récits des participants se limite au contenu manifeste plutôt qu'à son contenu latent. 


\section{4. Évaluation des variables psychologiques}

Afin de tenter de corroborer les données issues de l'entretien, nous avons procédé par triangulation des données et des méthodes, en utilisant des outils standardisés pour évaluer un certain nombre de variables psychologiques. La triangulation a pour objectif d'assurer une meilleure validité des données et de pouvoir accroître la compréhension du phénomène observé, notamment par le croisement de différentes sources de données [26].

Les échelles suivantes validées en français ont été utilisées :

- la LOT-R (Revised Life Orientation Test [25] pour évaluer l'optimisme ;

- le NEO-PI R (Revised NEO Personality Inventory) [19] pour évaluer le névrosisme ;

- la MAC (Mental Adjustement to Cancer Scale) [7] pour évaluer les stratégies de coping ;

- la HAD-S (Hospital Anxiety Depression Scale) [18] pour évaluer la symptomatologie dépressive et anxieuse ;

-1'EORTC-QLQC-30 [1] pour évaluer la qualité de vie.

\section{Analyses}

\subsection{Analyses qualitatives}

Sur les 37 patientes interviewées, 34 ont pu donner leur avis sur la campagne, les trois autres participantes n'ayant pas vu les spots télévisuels. Les entretiens intégralement retranscrits ont été analysés à l'aide du logiciel NVivo8 (QSR International). Afin de s'assurer la validité et la fidélité de l'analyse, une double codification de $15 \%$ du matériel de codage a été faite, une fois par le même codeur, puis par un second codeur. Cela s'est traduit par un taux de fidélité intra-juge de $83 \%$ et de fidélité inter-juges de $85 \%$. Ces coefficients de corrélation sont considérés comme élevés [4].

\subsection{Analyses quantitatives}

Des analyses de régressions multiples hiérarchiques ont été menées afin d'expliquer les changements de la qualité de vie et de l'état émotionnel des patientes au cours des quatre premiers mois de la maladie. Le premier bloc des prédicteurs inclut la valeur initiale de la variable dépendante (dimension de la qualité de vie, anxiété ou dépression à T0). Le 
deuxième bloc comprend les variables médicales ou sociodémographiques qui prédisent significativement la VD et les scores d'optimisme et de névrosisme. Les scores à l'échelle de coping constituent le troisième bloc.

\section{Résultats}

\subsection{Thématiques dégagées par l'analyse qualitative}

L'analyse des entretiens permet de souligner les processus identificatoires en jeu par rapport à la figure du héros ordinaire (tableau 2).

\subsubsection{Identification au héros (19/34, $55.8 \%)$}

On repère une utilisation répétée des termes en lien avec le lexique militaire (28/34, $82,3 \%)$ : combat, guerre, lutte, ne pas lâcher, ne pas se laisser envahir, ennemis. Ce sousgroupe fait référence à la position héroïque qu'elles se doivent de tenir. Des éléments tels la volonté, le sentiment d'efficacité perçue sont mis en exergue. Ainsi, la patiente $n^{\circ} 4$ dit: «Moi, je ne craquerai pas, y en a qui se plaignent [...] mais moi, ça m'arrivera pas, cette guerre, je vais la gagner. » On peut ainsi repérer l'importance des mécanismes de répression émotionnelle et du sentiment de contrôle (26/34, 76,4\%). Ainsi, les propos de la patiente $\mathrm{n}^{\circ} 17$ : «Après ma première chimio, je me suis arrêtée six fois sur la route pour vomir, mais après ça allait [...] Personne ne doit se rendre compte [...] J'ai un peu l'impression d'être une combattante, une guerrière, et ça, ça me donne du courage. » En outre, le psychisme est mis en avant comme un élément à part entière dans la guérison (22/34, 64,7\%). Les croyances profanes sur la nécessité de témoigner d'un état de bien-être psychologique se retrouvent de façon majoritaire dans le discours de ces femmes.

\subsubsection{Adhésion au contenu «ordinaire » de l'expression « héros ordinaires » $(9 / 34,26.4 \%)$}

Les expressions : «Je ne me bats pas, je fais avec, je subis », émaillent le discours de ces femmes. Ainsi, dans les propos de la patiente $n^{\circ} 32$, la perte de contrôle et un faible niveau d'efficacité perçu semblent tangibles $(8 / 34,25,4 \%)$ : «Moi, je ne sens pas du tout héroïque. Je suis malade et quoi que je fasse, je suis malade. Je n'ai pas le choix de me 
soigner, faut le faire. \ On peut également constater que l'expression des sentiments de malêtre est mise en avant $(7 / 34,20,5 \%)$.

En outre, la banalisation sociale de la maladie semble vécue péniblement (7/34, 20,5\%). Ainsi, les propos de la patiente $\mathrm{n}^{\circ} 7:$ « Tout le monde me dit que c'est rien un cancer du sein... Ça va vite passer, il faut que je serre les dents. Moi, je ne suis pas d'accord, il y a des gens qui meurent... c'est pas une petite maladie. »

\subsubsection{Une troisième voie : le cancer, une entité dépourvue de sens ? $(6 / 34,17,8 \%)$}

Les propos que nous avons rassemblés dans ce thème témoignent d'une impossible identification à l'expression «héros ordinaires » $(6 / 34,17,8 \%)$. Les femmes expriment une certaine incompréhension (patientes 33, 24, 12, 15) : "C'est ni l'un ni l'autre », "Ils n'ont vraiment pas l'air malades, ces gens, je ne vois pas le rapport avec ce que je vis ». Les opinions véhiculées font état d'une expérience de la maladie comparable à un deuil, un divorce, une perte d'emploi (5/34, 14,7\%). Ainsi, la patiente n 33 dit : «Quand j'ai divorcé, on ne m'a pas donné la Légion d'honneur, et là, je deviendrai héroïque car j'ai une maladie qui fait peur à tout le monde [...] J'ai perdu de la légèreté, comme quand je me suis retrouvée seule avec mes enfants, j'avais perdu ma sécurité de base. » Ces femmes évoquent les pertes réelles et symboliques auxquelles l'expérience de la maladie les expose. On repère également des mécanismes psychiques de mise en sens de l'événement traumatique dans une recherche de croissance personnelle $(5 / 34,14,7 \%)$.

\subsection{Triangulation des données qualitatives et quantitatives}

Afin de corroborer les données qualitatives, nous avons cherché à déterminer par régression multiple hiérarchique, pour l'ensemble de l'échantillon $(n=132)$, les prédicteurs de la qualité de vie et de la symptomatologie anxio-dépressive dans les quatre mois suivant la chirurgie. Les patientes ayant participé aux entretiens qualitatifs ne sont pas significativement différentes à T0 pour la qualité de vie totale $(\mathrm{t}(130)=1.34 ; \mathrm{p}=0.19)$, les scores de névrosisme $(\mathrm{t}(129)=0.001 ; \mathrm{p}=0.99)$, d'optimisme $(\mathrm{t}(130)=0.81 ; \mathrm{p}=0.41)$ le score total de symptomatologie anxiodépressive $(\mathrm{t}(130)=0.11 ; \mathrm{p}=0.92)$, et à $\mathrm{T} 1$, pour les préoccupations anxieuses $(\mathrm{t}(1128)=0.25 ; \mathrm{p}=0.80)$, l'esprit combatif $(\mathrm{t}(128)=0.33 ; \mathrm{p}=0.74)$, l'impuissance désespoir $(\mathrm{t}(128)=0.28 ; \mathrm{p}=0.78)$, l'évitement $(\mathrm{t}(128)=0.81 ; \mathrm{p}=0.42)$, et la dénégation $(\mathrm{t}(128)=0.14 ; \mathrm{p}=0.87)$. Les résultats présentés dans le tableau 3 montrent que les valeurs 
initiales entrées en bloc 1 prédisent significativement les variables dépendantes. Entrées au second pas, les variables sociodémographiques et médicales prédisent la qualité de vie physique, les activités, la qualité de vie sociale et la fatigue. Les variables de personnalité entrées au pas trois ne prédisent pas significativement les variables d'ajustement à quatre mois. Entrées au dernier bloc, les stratégies de coping ne prédisent que la variation de l'anxiété. Les femmes qui présentent des niveaux élevés de préoccupations anxieuses vis-àvis du cancer un mois après le diagnostic ont un niveau élevé d'anxiété quatre mois après la chirurgie $(\mathrm{F}=0,23, \mathrm{p}<0,05)$.

\section{Discussion}

Les patientes interrogées semblent ne pas pouvoir donner de réponse univoque à cette injonction paradoxale [27] que représenterait l'oxymore du héros ordinaire. Dans cette crise identitaire générée par la maladie, la possible identification à la figure du héros pourrait permettre d'évacuer temporairement le risque de s'assimiler à une communauté de malades, les autres. Ainsi, faire état de ses souffrances ne pourrait-il pas être considéré comme une mise en échec de cette identité de héros? On peut aussi s'interroger sur le risque d'une double stigmatisation [11] véhiculée par ce type de discours : un risque d'exclusion sociale déjà présent et fréquemment rapporté par les malades et une exclusion de celui qui ne s'identifierait pas au « combattant »?

On peut également repérer le décalage entre ce qui émerge du discours, notamment concernant une question qui ne parle pas directement de la participante et les résultats quantitatifs. Dans cette étude, les résultats font apparaître une absence de lien entre coping et ajustement émotionnel chez ces patientes, ce qui ne semble pas être le cas dans les entretiens. Cela peut s'expliquer par le fait que le processus de coping est complexe. Ainsi, il conviendrait de prendre en compte la flexibilité des stratégies de coping. La capacité de changer de stratégies de coping en fonction des exigences de la situation pourrait mieux rendre compte de l'ajustement psychologique des sujets [8].

\section{Conclusion}

Bien que l'on ne puisse affirmer de façon formelle que ce type de campagne a des effets dommageables sur les malades, il serait intéressant d'explorer plus avant les effets possibles d'un discours adressé au grand public sur les patients en rechute. Afin de faire 
évoluer les représentations sociales de la maladie cancéreuse et de la traiter symboliquement au même plan que toute autre forme de maladie chronique potentiellement létale, il conviendrait de s'interroger sur l'abandon bénéfique de la métaphore militaire [23].

\section{Conflits d'intérêts :}

- M. Barrault: Essai clinique en qualité de collaborateur à l'étude pour l'INCa et les laboratoires Lilly.

- G. Michel : Aucun

- F. Cousson-Gélie : Essai clinique en qualité d'investigateur principal à l'étude pour l'INCa et les laboratoires Lilly.

\section{Références}

[1] Aaronson NK, Ahmedzai S, Bergman B, et al. The European Organization for Research and Treatement of Cancer QLQ-C30: A Quality-of-Life Instrument for Use in International Clinical Trials in Oncology. Journal of National Cancer Institute 1993;85:365-75.

[2] Abuerri MF, Bataille P, Bobin JY, Dilhuydy JM, Feminier M, Genot PY, et al. Ensemble parlons autrement du cancer (Epac). Paris: Editions Aventis; 2001.

[3] American Cancer Society. Breast Cancer Facts \& Figures 2009-2010. Atlanta: American Cancer Society Inc.; 2009.

[4] Babcock JC, Miller SA, Siard C. Toward a typology of abusive women: Differences between partner-only and generally violent women in the use of violence. Psychology of Women Quarterly 2003;27:153-61.

[5] Beck F, Peretti-Watel P, Gautier A, Guilbert P. Regards sur le cancer : représentations et attitudes du public. Évolution 2006;4:1-6.

[6] Carver CS, Antoni MH. Finding benefit in breast cancer during the year after diagnosis predicts better adjustment 5 to 8 years after diagnosis. Health Psychology 2004;23:595-8.

[7] Cayrou S, Dickes P, Gauvain-Piquard A, Roge B. The mental adjustment to cancer (MAC) scale: French replication and assessment of positive and negative adjustment dimensions. Psychooncology 2003;12:8-23.

[8] Cheng C. Cognitive and motivational processes underlying coping flexibility: A dualprocess model. J Pers Soc Psychol 2003;84:425-38.

[9] Compas BE, Luecken L. Psychological adjustment to cancer. Current Directions in Psychological Science 2002;11:111-4.

[10] Étude barométrique INCa/Ipsos sur les représentations du cancer, point 1, janvier 2007. 
[11] Goffman E. Stigmate, les usages sociaux des handicaps. Paris: Éditions de Minuit; 1975.

[12] Hack TF, Degner LF. Coping responses following breast cancer diagnosis predict psychological adjustment three years later. Psycho-Oncology 2004;13:235-47.

[13] Institut de Veille Sanitaire. Estimation de l'incidence et de la mortalité par cancer en France de 1980 à 2005, 2009. Available from: URL: http://www.invs.sante.fr/surveillance/cancers/estimations_cancers/default.htm.

Accessed december 2009.

[14] Institut National du Cancer. Campagne 2007 «Nous sommes deux millions de héros ordinaires ». Available from URL : http://www.e-cancer.fr/videos/videos-des-heros-ordinaires. Accessed september 2008.

[15] Miles MB, Huberman AM. Qualitative data analysis: an expanded sourcebook (2nd ed.). Thousand Oaks: Sage Publications; 1994.

[16] National Coalition for Cancer Survivorship (NCCS). Self-advocacy. A cancer survivor's handbook. Available from URL : http://www.canceradvocacy.org/resources/publications/advocacy.pdf. Accessed December 07, 2009.

[17] Pirl WF. Evidence report on the occurrence, assessment, and treatment of depression in cancer patients. J Natl Cancer Inst Monogr 2004;32:32-9.

[18] Razavi D, Delvaux N. Psycho-Oncologie : le cancer, le malade et sa famille. Paris: Masson; 2002.

[19] Rolland JP, Petot JM. NEO PI-R, Inventaire de Personnalité-Révisé. Paris: Éditions du Centre de Psychologie Appliquée; 1998.

[20] Roy-Byrne PP, Davidson KW, Kessler RC, et al. Anxiety disorders and comorbid medical illness. Gen Hosp Psychiatry 2008;30:208-25.

[21] Schmidt JE, Andrykowski MA. The role of social and dispositional variables associated with emotional processing in adjustment to breast cancer: An internet-based study. Health Psychology 2004;23:259-66.

[22] Sears SR, Stanton AL, Danoff-Burg S. The yellow brick road and the emerald city: Benefit-finding, positive reappraisal coping, and posttraumatic growth in women with earlystage breast cancer. Health Psychology 2003;22:487-97.

[23] Sontag S. Illness as metaphor. New York: Farrar, Straus \& Giroux; 1978.

[24] Stark D, Kiely M, Smith A, Velikova G, House A, et al. Anxiety disorders in cancer patients: Their nature, associations, and relation to quality of life. J Clin Oncol 2002;20:313748. 
[25] Sultan S, Bureau B. Which optimism in health psychology? European Review of Applied Psychology 1999;49:43-51.

[26] Van Der Maren JM. Méthodes de recherche pour l'éducation. Bruxelles: De Boeck et Laurcier; 1996.

[27] Watzlawick P, Beavin-Bavelas J, Jackson D. Une logique de la communication. Paris: Seuil; 1972. 


\section{Tableau 1}

Caractéristiques socio-démographiques et médicales de l'échantillon $(\mathrm{N}=37)$

\begin{tabular}{lc}
\hline & $\begin{array}{c}\text { Moyenne (écart-type) ou } \\
\text { pourcentage }\end{array}$ \\
\hline Age & $54.87(11.45)$
\end{tabular}

Situation familiale:

Vie seule

séparée

Vie maritale

Niveau d'études

Pas d'études

CAP-BEP

Baccalauréat

$\mathrm{BAC}+2$

$\mathrm{BAC}+3$ et plus

Engagement professionnel

Sans profession

En activité

En recherche d'emploi

Retraité

Troubles psychologiques antérieurs

Caractéristiques médicales

T0

T1

T2

$\mathrm{T} 3$

T4

N0

N1

N2

N3

Grade histologique

Grade 1
1.3

53.1

18.9

18.9

24.3

5.4

70.5

8.1

29.7

18.9

18.9

2.9

68.9

23.3

3.9

1

62.7

30.4

4.9

2

26.5 
Grade 2

Grade 3

Type de chirurgie

Tumorectomie

Mastectomie

Traitements adjuvants

Chimiothérapie

Radiothérapie

Hormonothérapie

Herceptine

10.8

\section{Tableau 2}

Thèmes principaux et secondaires abordés par les femmes interrogées sur leurs perceptions de la campagne de sensibilisation «Nous sommes deux millions de héros ordinaires» (fréquence et pourcentage de femmes).

1. Identification à la figure du héros

2. Adhésion au contenu «ordinaire» de l'expression
1.1 Recours au lexique militaire 1.2 Importance des mécanismes de répression émotionnelle et du sentiment de contrôle 1.3 Rôle central du psychisme dans le processus de guérison

2.1 Perte de contrôle et faible sentiment d'efficacité perçue

2.2 Valorisation de l'expression des affects négatifs

2.3 Refus de la banalisation sociale de la maladie

3.1 Une identification impossible

3.2 La maladie, un événement de vie sous le sceau de la perte

3.3 Mise en sens de l'événement traumatique sans recherche de causalité
$19 / 34 ; 55.8 \%$

$28 / 34 ; 82.3 \%$

$26 / 34 ; 76.4 \%$

$22 / 34 ; 64.7 \%$

$9 / 34 ; 26.4 \%$

$8 / 34 ; 25.4 \%$

$7 / 34 ; 20.5 \%$

$7 / 34 ; 20.5 \%$

$6 / 34 ; 17.8 \%$

$6 / 34 ; 17.8 \%$

$5 / 34 ; 14.7 \%$

$5 / 34 ; 14.7 \%$ 
Tableau 3

Régression multiple hiérarchique sur les variables de qualité de vie et d'ajustement psychologique 4 mois après la chirurgie.

\begin{tabular}{|c|c|c|c|c|c|}
\hline $\begin{array}{l}\text { Variable } \\
\text { dépendante }\end{array}$ & $\begin{array}{l}\text { Blocs de } \\
\text { prédicteurs }\end{array}$ & $\overline{\mathrm{R}^{2}}$ & $\overline{\mathrm{R}^{2}}$ & ddl & $\vec{F}$ \\
\hline Qualité de vie & 1 & 0.11 & 0.11 & 1.120 & $15.11 * * *$ \\
\hline \multirow[t]{3}{*}{ globale } & 2 & 0.05 & 0.16 & 3.117 & 2.32 \\
\hline & 3 & 0.01 & 0.17 & 2.115 & 0.78 \\
\hline & 4 & 0.06 & 0.24 & 5.110 & 1.77 \\
\hline \multirow[t]{4}{*}{ QDV physique } & 1 & 0.13 & 0.13 & 1.120 & $17.64 * * *$ \\
\hline & 2 & 0.07 & 0.20 & 3.117 & $3.37 *$ \\
\hline & 3 & 0.01 & 0.20 & 2.115 & 0.33 \\
\hline & 4 & 0.02 & 0.22 & 5.110 & 0.56 \\
\hline \multirow[t]{4}{*}{ Activités } & 1 & 0.06 & 0.06 & 1.120 & $7.30 * *$ \\
\hline & 2 & 0.10 & 0.16 & 3.117 & $4.83 * *$ \\
\hline & 3 & 0.01 & 0.17 & 2.115 & 0.24 \\
\hline & 4 & 0.01 & 0.18 & 5.110 & 0.33 \\
\hline QDV & 1 & 0.12 & 0.12 & 1.120 & $16.57 * * *$ \\
\hline \multirow[t]{3}{*}{ émotionnelle } & 2 & 0.02 & 0.14 & 3.117 & 0.77 \\
\hline & 3 & 0.00 & 0.14 & 2.115 & 0.21 \\
\hline & 4 & 0.03 & 0.17 & 5.110 & 0.81 \\
\hline \multirow[t]{4}{*}{ QDV sociale } & 1 & 0.12 & 0.12 & 1.120 & $16.74 * * *$ \\
\hline & 2 & 0.11 & 0.23 & 3.117 & $5.52 * *$ \\
\hline & 3 & 0.01 & 0.24 & 2.115 & 0.52 \\
\hline & 4 & 0.01 & 0.25 & 5.110 & 0.40 \\
\hline \multirow[t]{4}{*}{ Fatigue } & 1 & 0.07 & 0.07 & 1.120 & $8.52 * *$ \\
\hline & 2 & 0.10 & 0.17 & 3.117 & $4.94 * *$ \\
\hline & 3 & 0.01 & 0.18 & 2.115 & 0.68 \\
\hline & 4 & 0.00 & 0.18 & 5.110 & 0.08 \\
\hline \multirow[t]{3}{*}{ Anxiété } & 1 & 0.22 & 0.22 & 1.120 & $34.05 * * *$ \\
\hline & 2 & 0.00 & 0.23 & 3.117 & 0.23 \\
\hline & 3 & 0.00 & 0.23 & 2.115 & 0.35 \\
\hline
\end{tabular}




\begin{tabular}{l|lllll} 
& 4 & 0.09 & 0.32 & 5.110 & $2.79^{*}$ \\
Dépression & 1 & 0.21 & 0.21 & 1.120 & $32.27^{* * *}$ \\
2 & 0.03 & 0.24 & 3.117 & 1.37 \\
3 & 0.00 & 0.24 & 2.115 & 0.11 \\
& 4 & 0.05 & 0.29 & 5.110 & 1.60 \\
\hline
\end{tabular}

${ }^{*} \mathrm{p}<0.05 * * \mathrm{p}<0.01 * * \mathrm{p}<0.001$

\section{Discussion}

Dr Tésu-Rollier - Merci de la présentation très intéressante que vous venez de faire sur un thème peu exploré et d'avoir pu souligner clairement l'intérêt de croiser des approches qualitatives et quantitatives afin de mieux cerner cette problématique qui renvoie à un questionnement intime et sociétal. Je souhaiterais savoir sur quels types d'instances scientifiques et/ou de communication, l'INCa s'est appuyé pour construire cette campagne?

Réponse du rapporteur, Mme Barrault - Merci de vos remarques et de cette intéressante question. Les informations que je peux vous fournir en ce qui concerne spécifiquement la campagne «Nous sommes deux millions de héros ordinaires » ont été recueillies sur le site institutionnel de l'INCa. On apprend ainsi que la campagne a été organisée par une agence de communication, non spécialisée dans le domaine de la santé, et par une agence de production audiovisuelle, chargée notamment de sélectionner les individus, « les vrais gens », susceptibles de vouloir témoigner de leurs histoires « avec émotion ». À ce jour, je ne suis pas en mesure de vous dire si une évaluation de l'impact de cette campagne plus exhaustive que celle de l'étude que je viens de vous présenter a pu être réalisée.

Cependant, probablement dans un souci d'évaluation, l'INCa a lancé une étude pluriannuelle sur l'observation et l'analyse des représentations sociales du cancer à travers les discours médiatiques et l'opinion publique en ligne. Cette étude, menée notamment par le Ceditec (Centre d'étude des discours, images, textes, écrits, communications, Université Paris 12), a été réalisée sur la base exhaustive des articles de presse et des programmes de radio et de télévision consacrés au cancer entre janvier 2008 et décembre 2008. On peut regretter que pour le moment, l'analyse des productions des institutions publiques en matière 
de santé semble peu croiser avec une analyse du vécu des individus malades exposés à ce type de discours. 